\title{
Relación entre los factores de riesgo cardiovascular y el patrón de aterosclerosis de miembros inferiores en pacientes sometidos a angioplastia
}

\section{Relationship between cardiovascular risk factors and the pattern of lower limb atherosclerosis in patients undergoing angioplasty}

\author{
Miguel G. Carrizales-Vázquez, Óscar E. Reyes-Aguirre, Carlos A. Ávila-Morales y Alberto C. Robles-Solís*
}

Departamento de Angiología, Cirugía Vascular y Endovascular, Unidad Médica de Alta Especialidad, Hospital de Especialidades No. 71, Torreón, Coahuila, México

\begin{abstract}
Resumen
Antecedentes: La causa más común de enfermedad arterial periférica (EAP) es la aterosclerosis y sigue ciertos patrones de distribución de acuerdo con los factores de riesgo cardiovascular. Objetivo: Determinar el patrón de distribución aterosclerótico en extremidades inferiores que se vincula con ciertos factores de riesgo cardiovascular regulares en esta unidad. Material y métodos: Estudio transversal y retrolectivo en el que se determinó la relación de las variables examinadas con la prueba $\chi^{2}$ de Pearson y razón de momios para prevalencia (RMP) para dilucidar si un factor de riesgo es independiente; se realizaron modelos de regresión logística multivariable ajustados por los factores de riesgo estudiados. Resultados: Los pacientes con DM se vincularon con enfermedad de vasos tibiales $(p<0.001)$; RMP ajustado (2.473 [1.190-5.137]; $n=145) ;$ el segmento aortoilíaco se relaciona con el tabaquismo (RMP, 3.685 [1.346-10.087]) y al final con el segmento femoropoplíteo; el género femenino se vincula más que el masculino $(p=0.022)$, con RMP ajustado para hombres: 0.466 (0.253-0.862), $n=73$. Conclusiones: Los factores de riesgo cardiovascular de cada paciente ayuda a conocer el patrón de distribución aterosclerótico con el que se relacionan. Los pacientes con DM muestran enfermedad de vasos tibiales, el género femenino se acompaña de enfermedad femoropoplítea y el tabaquismo con enfermedad aortoilíaca en esta población de estudio.
\end{abstract}

Palabras clave: Aterosclerosis de miembros inferiores. Enfermedad arterial periférica. Diabetes mellitus. Patrón arteriográfico de extremidades inferiores. Factores de riesgo cardiovascular.

\begin{abstract}
Background: The most common cause of peripheral artery disease (PAD) is atherosclerosis, and it follows distribution patterns according to the cardiovascular risk factor. Objective: To determine the atherosclerotic distribution patterns in the lower extremities in association to specific cardiovascular risk factors frequent in our unit. Methods: Pearson's chi-squared test and Odds Ratio for prevalence (ORP) were used to determine the association of the variables. To determine whether Diabetes Mellitus (DM) is an independent risk factor for the distribution pattern, we performed multivariate logistic regression models adjusted for the studied cardiovascular risk factors. Results: Patients with DM are associated with tibial artery disease
\end{abstract}

Correspondencia:

*Alberto C. Robles-Solís

E-mail: alberto190991@gmail.com

$0377-4740 / 02021$ Sociedad Mexicana bajo la licencia CC BY-NC-ND license (http://creativecommons.org/licenses/by-nc-nd/4.0/).
Available online: 15-04-2021 Rev Mex Angiol. 2021;49(1):8-15 www.RMAngiologia.com 
$(P<0.001)$ adjusted ORP: $(2.473$ [1.190-5.137] $n=145)$. Aortoiliac disease is associated with smoking (ORP 3.685 [1.34610.087]) and hypertension is les associated in this segment ORP: 0.143 (0.061-0.334). In the femoropopliteal segment, male sex is associated less ORP 0.466 (0.253-0.862), $n$ : 73. Conclusions: Each patient's cardiovascular risk factors help us by knowing the atherosclerotic distribution pattern with which it's associated: diabetic patients are associated with tibial artery disease, females are associated with femoropopliteal disease, and smoking with aortoiliac disease in our studied population. Key words: Lower extremities atherosclerosis. Peripheral artery disease. Diabetes Mellitus. Arteriography patterns in the lower extremities. Cardiovascular risk factors.

Key words: Lower extremities atherosclerosis. Peripheral artery disease. Diabetes Mellitus. Arteriography patterns in the lower extremities. Cardiovascular risk factors.

\section{Introducción}

El término "enfermedad de la arteria periférica" abarca por lo regular las diversas enfermedades que afectan a las arterias no cardíacas y no intracraneales ${ }^{1}$. La enfermedad arterial periférica (EAP) de la extremidad inferior se refiere a una obstrucción parcial o completa de las arterias de las extremidades inferiores. La causa común es la aterosclerosis ${ }^{2}$. La EAP, después del infarto agudo de miocardio y el accidente cerebrovascular, es la tercera causa principal de morbilidad y mortalidad cardiovascular aterosclerótica en todo el mundo ${ }^{3}$. En 2010, los cálculos sugirieron que $>200$ millones de personas en todo el mundo vivían con EAP. Esto representó un aumento del $23.5 \%$ respecto del 2000 , incremento que en gran medida se atribuye al envejecimiento de la población y la creciente prevalencia de factores de riesgo, en particular diabetes mellitus (DM) ${ }^{1,4-6}$.

Aunque el número de personas con EAP ha aumentado en la última década, en países de ingresos bajos o medios se han realizado escasos estudios epidemiológicos para establecer determinaciones confiables de prevalencia y distribución de factores de riesgo, en especial en América Latina, incluido México, donde las enfermedades cardiovasculares (ECV) se han convertido en la principal causa de muerte y discapacidad? Por lo tanto, aún se necesitan estudios para comprender mejor la etiología y la distribución de enfermedades, y también para desarrollar políticas y programas más eficaces para la prevención y tratamiento de la $\mathrm{EAP}^{3}$.

Los estudios existentes han confirmado que los factores de riesgo más importantes (diabetes, hipertensión, tabaquismo e hiperlipidemia) intervienen en el $80 \%$ a $90 \%$ de las enfermedades cardiovasculares ${ }^{5,8,9}$. La DM se relaciona con un riesgo de amputación 10 veces mayor que el de los pacientes no diabéticos ${ }^{8}$, es causa de enfermedad oclusiva predominantemente distal, incluidas las arterias tibiales y pedias, y afecta a las arterias de las extremidades de dos maneras: al promover la enfermedad oclusiva aterosclerótica y al endurecer la pared arterial relacionada con la calcinosis medial $^{2}$. Se ha demostrado un nexo entre DM y EAP; el riesgo de claudicación intermitente es casi el doble en pacientes con diabetes respecto de personas sin diabetes $^{10}$. La Federación Internacional de Diabetes (FID) ha elaborado cálculos de la prevalencia de diabetes desde el año 2000 y ha publicado que 578 millones de adultos tendrán diabetes en el año 2030, cifra que alcanzará los 700 millones para 2045, y de éstos una tercera parte presentará algún grado de enfermedad arterial periférica ya que los estudios recientes han proyectado una prevalencia de $30.7 \%$ de EAP entre los diabéticos ${ }^{11,12}$.

Dentro de la bibliografía más reciente, el tabaquismo tiende a relacionarse más con la participación de la EA (enfermedad aortoilíca) y la FP (femoropoplítea), mientras que la diabetes posee un sólido nexo con los patrones patológicos de la región FP y vasos tibiales ${ }^{4,13}$. Este estudio tiene como objetivo determinar qué factor es predominante en las lesiones ateroscleróticas en las distintas regiones estudiadas y comparar estos resultados con las publicaciones actuales internacionales y obtener estadísticas locales y nacionales.

\section{Material y métodos}

\section{Diseño del estudio y participantes}

Estudio transversal retrolectivo que recabó de las variables de expedientes clínicos físicos y electrónicos los resultados de las arteriografías diagnósticas terapéuticas realizadas en el período comprendido entre el 1 de enero del 2018 y el 31 de marzo del 2020 en la Unidad Médica de Alta Especialidad HE 71 (UMAE 71), Torreón, Coahuila, una vez aprobado el protocolo por el comité de ética e investigación de esta unidad. Los pacientes procedieron del servicio de Angiología y Cirugía Vascular del 
UMAE 71 , una de cuyas consideraciones más importantes es que es de referencia para los estados de Durango, Zacatecas, Chihuahua y Coahuila. Los pacientes elegibles fueron aquéllos con algún grado de enfermedad aterosclerótica de miembros inferiores corroborado por algún estudio de imagen (ultrasonido Doppler, angiografía por resonancia magnética, angiotomografía) o que ya acudieran con arteriografía a consulta externa y tuvieran algún factor de riesgo cardiovascular; los incluidos en este estudio fueron diabetes mellitus, hipertensión arterial sistémica, enfermedad renal crónica y cardiopatía isquémica, y los individuos se sometieron a arteriografía diagnóstica/terapéutica en esta unidad en el periodo ya referido. Entre los criterios que se consideraron para excluir al paciente del estudio fue tener expediente incompleto, en particular que no contara con historia clínica completa y hoja de informe de arteriografía por esta unidad o que éste indicara un informe incompleto, esto es, que no se notificaran los hallazgos de los segmentos aortoilíacos, femoropoplíteos y de vasos tibiales. Para el tamaño de muestra se utilizó la fórmula para diferencias de proporciones y se permitió un error $<0.05(n=168)$.

\section{Metodología de la recolección de datos}

Se recolectaron los datos de acuerdo con las variables estudiadas comentadas con anterioridad. La ubicación de las lesiones blanco se agrupó como aortoilíaca o proximal (aorta, arteria ilíaca común, externa e interna), femoropoplítea (arterias femorales comunes, superficiales y profundas, arteria poplítea) e infragenicular 0 enfermedad de vasos tibiales (tronco tibioperoneo, arteria tibial anterior y posterior, arteria peronea hasta el arco plantar). Se consideró como lesión significativa > $50 \%$ de estenosis del vaso. Los factores de riesgo incluidos en el modelo estadístico fueron edad (en años y como medida de tendencia central la media) y género; el estadio clínico se dividió en cuatro grados por la escala clínica de Fontaine; el diagnóstico de diabetes mellitus se definió como cifras de glucosa y hemoglobina glucosilada acordes a los criterios diagnósticos ADA 2019; la hipertensión arterial se definió por presión arterial sistólica $\geq 130 \mathrm{mmHg}$ o presión arterial diastólica $\geq 80 \mathrm{mmHg}$, según lo establecieron la ACC/AHA, o bien si el paciente consumió algún medicamento antihipertensivo. Los pacientes se clasificaron como fumadores y no fumadores, de acuerdo con la entrevista con el paciente o lo documentado en la historia clínica. Se tomaron en cuenta a los pacientes con diagnóstico de enfermedad renal crónica, los individuos con antecedentes de tratamiento sustitutivo renal por diálisis o hemodiálisis, esto es, una depuración de creatinina $<15 \mathrm{ml} / \mathrm{min} / 1.73 \mathrm{~m}^{2}$ o aquellos que contaran con dicho diagnóstico ya conformado por una nota del servicio de nefrología, y por último los pacientes con antecedente de cardiopatía isquémica confirmada por el servicio de cardiología y los enfermos ya con seguimiento por dicho servicio y lo informado en su expediente clínico.

\section{Análisis estadístico}

Este estudio se diseñó para identificar qué factor de riesgo se relaciona en grado significativo en cada segmento arterial. La muestra se conformó con 229 pacientes. Se determinó la relación de las variables examinadas con la prueba $\chi^{2}$ de Pearson, así como la razón de momios (RM) para valorar la probabilidad estadísticamente significativa entre el factor de riesgo-enfermedad y se consideró una significancia estadística como $p<0.05$; con posterioridad, para dilucidar si algún factor de riesgo era independiente para el patrón de distribución, se realizaron modelos de regresión logística multivariable ajustados por los factores de riesgo cardiovascular estudiados.

\section{Aspectos éticos}

El estudio se sometió al comité de ética e investigación de la Unidad Médica de Alta Especialidad HE 71 en Torreón, Coahuila, y se efectuó apegado a los principios científicos y éticos previstos en la Ley General de Salud de México en materia de investigación, así como acorde a la declaración de Helsinki y a los códigos y normas internacionales vigentes de las buenas prácticas de la investigación. Los datos personales de cada paciente se mantendrán en confidencialidad. Los datos obtenidos del estudio no afectarán las decisiones en los pacientes, por lo que no se requirió consentimiento informado. Este estudio no contó con algún tipo de financiamiento, los gastos corrieron por cuenta de los investigadores.

\section{Resultados \\ Datos generales}

Se incluyó a 281 pacientes del período comprendido entre el 1 de enero del 2018 y el 31 de marzo del 2020 y se recabaron datos de bitácora de sala de hemodinamia, archivo clínico y archivo electrónico de la institución; de éstos sólo 229 cumplían los criterios de inclusión de este estudio y el resto se excluyó por los criterios ya descritos. Se tomó en cuenta la extremidad por la cual 
Tabla 1. Características demográficas, factores de riesgo y distribución general de la enfermedad arterial periférica de las extremidades inferiores en pacientes diabéticos y no diabéticos de la UMAE 71 atendidos en el periodo de enero de 2018 a marzo de 2020

\begin{tabular}{|c|c|c|}
\hline Variable & Diabéticos ( $n=184$ ) & No diabéticos ( $n=45$ ) \\
\hline Edad (años), media (DE) & $66(10.4)$ & 70 (10.5) \\
\hline Hombres & $90(48.9)$ & $29(64.4)$ \\
\hline $\begin{array}{l}\text { Localidad de origen } \\
\text { Chihuahua } \\
\text { Coahuila } \\
\text { Durango } \\
\text { Zacatecas }\end{array}$ & $\begin{array}{l}66(35.9) \\
52(28.3) \\
38(20.7) \\
28(15.2)\end{array}$ & $\begin{array}{l}17(37.8) \\
14(31.1) \\
8(17.8) \\
6(13.3)\end{array}$ \\
\hline $\begin{array}{l}\text { Factores de riesgo cardiovascular } \\
\text { Tabaquismo } \\
\text { Hipertensión arterial } \\
\text { Enfermedad renal crónica } \\
\text { Cardiopatía isquémica }\end{array}$ & $\begin{array}{c}81(44) \\
141(76.6) \\
33(17.9) \\
31(16.8)\end{array}$ & $\begin{array}{c}36(80) \\
31(68.9) \\
3(6.7) \\
13(28.9)\end{array}$ \\
\hline $\begin{array}{l}\text { Distribución anatómica de la enfermedad aterosclerótica } \\
\text { Enfermedad aortoilíaca } \\
\text { Enfermedad femoropoplítea } \\
\text { Enfermedad de vasos tibiales } \\
\text { Extremidad inferior izquierda } \\
\text { Extremidad inferior derecha }\end{array}$ & $\begin{array}{l}17(9.2) \\
120(65.2) \\
145(78.8) \\
85(46.6) \\
85(46.2)\end{array}$ & $\begin{array}{l}22(48.9) \\
36(80) \\
23(51.1) \\
17(37.8) \\
9(20)\end{array}$ \\
\hline $\begin{array}{l}\text { Estadio clínico prequirúrgico } \\
\text { Fontaine IIB } \\
\text { Fontaine III } \\
\text { Fontaine IV }\end{array}$ & $\begin{array}{c}30(16.3) \\
28(15.2) \\
126(68.5)\end{array}$ & $\begin{array}{l}15(33.3) \\
11(24.4) \\
18(40)\end{array}$ \\
\hline $\begin{array}{l}\text { Otras características relevantes de la población en estudio } \\
\text { Glucosa prequirúrgica (mg/dl), mediana (RIO) } \\
\text { Antecedente de diabetes mellitus en años, media (DE) }\end{array}$ & $\begin{array}{c}129(91-201) \\
19(8.6)\end{array}$ & $\begin{array}{c}92(80-116) \\
N A\end{array}$ \\
\hline
\end{tabular}

Los valores son el número de pacientes (porcentajes) a menos que se indique otra cosa

n: número de pacientes; DE: desviación estándar; RIQ: rango intercuartílico; NA: no aplica para este grupo.

acudieron a consulta, algo importante mencionar ya que se obtuvieron pocos mapeos de extremidad contralateral a la consultada. Se consideró sólo el primer procedimiento del paciente realizado en la unidad. Los factores de riesgo y datos generales se tomaron de la historia clínica y hoja de ingreso del expediente físico; si no era posible la obtención de datos fue necesario realizar el análisis del expediente electrónico del paciente hasta completar las variables a estudiar; cuando no se disponía de dicha información, se excluía a este paciente del estudio.

De los 229 pacientes totales, 119 fueron del sexo masculino (52.0\%) y 110 del femenino (48.0\%) y se observó que se atendió a más pacientes del estado de Chihuahua, con 83 (36.2\%). Del total de participantes, 184 (80.3\%) eran diabéticos y $45(19.7 \%)$ no diabéticos, pero con algún factor de riesgo de los estudiados (hipertensión, tabaquismo, cardiopatía isquémica, enfermedad renal crónica). Los hipertensos totales fueron 172 pacientes $(75.1 \%)$, los fumadores $117(51.1 \%)$, los sujetos con enfermedad renal crónica 36 (15.7\%) y los individuos con cardiopatía isquémica 44 (19.2\%). La edad media general fue de 66 años; la mayoría de los pacientes llegó a consulta en un estadio IV de Fontaine, 144 pacientes $(62.9 \%)$ del total (Tabla 1). De los diabéticos, la media de años con diagnóstico de diabetes mellitus fue de 19 y la mediana en cuanto a la glucosa antes del procedimiento fue de 129 (RIQ, 91-201) mg/dl (Tabla 1).

\section{Distribución anatómica de las lesiones ateroscleróticas}

La región más afectada del total de pacientes fue el segmento de vasos tibiales con 168 individuos (73.4\%) del total; esta área de la extremidad izquierda fue más afectada respecto de la derecha, con 102 pacientes $(60.7 \%)$. El segmento femoropoplíteo se vio comprometido en 156 individuos (68.1\%) del general y el segmento menos afectado fue el aortoilíaco, con 39 pacientes (17.0\%) (Tabla 1). 
Tabla 2. Resultados de la relación entre factores de riesgo cardiovascular estudiados y enfermedad aterosclerótica de las extremidades inferiores por segmentos en pacientes diabéticos y no diabéticos atendidos en la UMAE 71 en el periodo de enero de 2018 a marzo de 2020

\begin{tabular}{|c|c|c|c|}
\hline \multirow[t]{2}{*}{ Variable } & \multicolumn{3}{|c|}{ Enfermedad aortoilíaca } \\
\hline & & Relación $\chi^{2}$ & OR no ajustado \\
\hline Hombres & $26(66.7)$ & 0.044 & $2.08(1.01-4.30)$ \\
\hline Tabaquismo & $33(84.6)$ & $<0.001$ & $6.94(2.77-17.3)$ \\
\hline Hipertensión arterial & $28(71.8)$ & 0.599 & $0.81(0.37-1.76)$ \\
\hline Enfermedad renal crónica & $1(2.6)$ & 0.013 & $0.11(0.01-0.87)$ \\
\hline Cardiopatía isquémica & $6(15.4)$ & 0.505 & $0.72(0.28-1.86)$ \\
\hline \multirow[t]{3}{*}{ Diabetes mellitus } & $17(43.6)$ & $<0.001$ & $0.10(0.04-0.23)$ \\
\hline & \multicolumn{3}{|c|}{ Enfermedad femoropoplítea } \\
\hline & & Relación $\chi^{2}$ & OR no ajustado \\
\hline Hombre & $73(46.8)$ & 0.022 & $0.51(0.29-0.91)$ \\
\hline Tabaquismo & $78(50.0)$ & 0.629 & $0.87(0.50-1.52)$ \\
\hline Hipertensión arterial & $115(73.7)$ & 0.477 & $0.78(0.40-1.52)$ \\
\hline Enfermedad renal crónica & $24(15.4)$ & 0.839 & $0.92(0.43-1.96)$ \\
\hline Cardiopatía isquémica & $32(20.5)$ & 0.466 & $1.31(0.63-2.72)$ \\
\hline \multirow[t]{3}{*}{ Diabetes mellitus } & $120(76.9)$ & 0.056 & $0.46(0.21-1.03)$ \\
\hline & \multicolumn{3}{|c|}{ Enfermedad de vasos tibiales } \\
\hline & & Relación $\chi^{2}$ & OR no ajustado \\
\hline Hombres & $85(50.6)$ & $0.049 a$ & $0.81(0.45-1.4)$ \\
\hline Tabaquismo & $75(44.6)$ & 0.001 & $0.36(0.19-0.67)$ \\
\hline Hipertensión arterial & $131(78)$ & 0.096 & $1.72(0.90-3.29)$ \\
\hline Enfermedad renal crónica & $32(19)$ & 0.022 & $3.35(1.13-9.91)$ \\
\hline Cardiopatía isquémica & $29(17.3)$ & 0.213 & $0.64(0.31-1.29)$ \\
\hline Diabetes mellitus & $145(86.3)$ & $<0.001$ & $3.55(1.79-7.04)$ \\
\hline
\end{tabular}

Los valores son el número de pacientes (porcentajes) a menos que se indique otro valor. $\chi^{2}$ : prueba ji cuadrada de Pearson.

\section{Distribución de resultados entre la relación de variables y resultados significativos y no significativos entre factores de riesgo y afectación de cada sección anatómica}

Los resultados demuestran que los pacientes con DM se acompañan de enfermedad de vasos tibiales ( $p$ < 0.001; RMP, 3.556 [1.796-7.041]; RMP ajustado, 2.473 [1.190-5.137]; $n=145$ ) (Tabla 2), así como en pacientes con enfermedad renal crónica ( $p=0.022$; RMP, 3.35 [1.13-9.91]; $n=32$ ) (Tabla 3). Sin embargo, al realizar el ajuste de RMP mediante regresión logística
(RMP, 2.503 [0.816-7.67]), no se identificó significancia estadística, por lo cual se confirió una mayor relación y riesgo a la DM como factor de riesgo en este segmento (Fig. 1). En cuanto al tabaquismo, se encontró una menor relación ( $p<0.001$; RMP ajustado, 0.466 [0.232$0.0 .935] ; n=75)$ para enfermedad en este segmento (Tabla 4).

En el segmento femoropoplíteo, los resultados demuestran que el sexo femenino se vincula más con enfermedad respecto del sexo masculino $(p=0.022$; con RMP ajustado para hombres, 0.466 [0.253-0.862]; $n=73$ ) (Tabla 4). La hipertensión arterial se relacionó menos, con RMP ajustado de 0.415 (0.180-0.956). 
Tabla 3. Modelo de regresión logística para el desarrollo de enfermedad de vasos tibiales entre pacientes diabéticos y no diabéticos que tienen otro factor de riesgo cardiovascular de UMAE 71 en el periodo de enero de 2018 a marzo de 2020

\begin{tabular}{|l|c|c|c|c|}
\hline Variable & B & OR ajustado & \multicolumn{2}{|c|}{ IC95\% } \\
\cline { 2 - 5 } & & & Mínimo & Máximo \\
\hline Hombres & 0.175 & 1.191 & 0.613 & 2.315 \\
\hline Tabaquismo & -0.763 & 0.466 & 0.232 & 0.935 \\
\hline Hipertensión arterial & 0.470 & 1.601 & 0.789 & 3.246 \\
\hline Enfermedad renal crónica & 0.917 & 2.503 & 0.816 & 7.675 \\
\hline Cardiopatía isquémica & -0.520 & 0.595 & 0.275 & 1.287 \\
\hline Diabetes mellitus & 0.905 & 2.473 & 1.190 & 5.137 \\
\hline
\end{tabular}

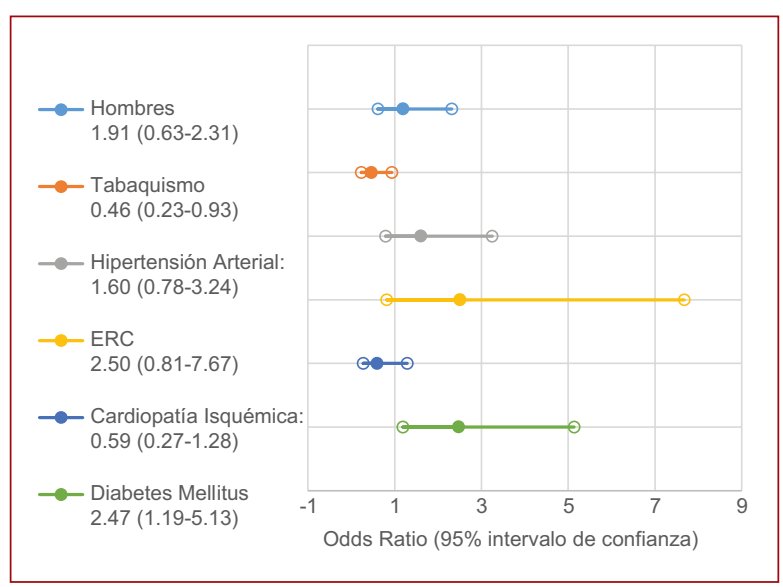

Figura 1. Resultado del modelo de regresión logística para el desarrollo de enfermedad de vasos tibiales entre pacientes diabéticos y no diabéticos que prueban la relación entre enfermedad de vasos tibiales y pacientes con diabetes mellitus atendidos en la UMAE 71 en el periodo de enero de 2018 a marzo de 2020.

En el segmento aortoilíaco, los resultados demuestran que los pacientes fumadores se vinculan significativamente con enfermedad en este segmento ( $p=0.044$; RMP ajustado, 3.685 [1.346-10.087]; $n=33$ ), así como ser hipertenso confiere menos nexo con enfermedad en este segmento (RMP ajustado, 0.143 [0.061-0.334]; $n=28$ ); el resto de los factores mostró resultados no significativos (Tabla 4 ).

\section{Discusión de resultados}

Estudio transversal retrolectivo realizado en un solo centro en el norte de México cuyos resultados demuestran que existe una relación significativa entre la enfermedad de vasos tibiales en pacientes con DM en comparación con los no diabéticos, pero con otro factor de riesgo cardiovascular. Aunque la enfermedad renal crónica mostró resultado significativo con razón de momios no ajustada en este segmento, al realizar modelos de regresión logística y ajustar la razón de momios no se observó significancia estadística. Esto permite inferir que hay otros factores que contribuyen en estos paciente a la enfermedad de vasos tibiales y una de las posibles causas es que en México el origen más frecuente de enfermedad renal crónica es la DM ${ }^{14}$. En el segmento aortoilíaco, el tabaquismo mostró significancia estadística como factor de riesgo relacionado y la hipertensión arterial reveló que se vincula menos en este segmento. $Y$ en el segmento femoropoplíteo, el sexo femenino señaló una relación significativa para la enfermedad y, al igual que en el segmento aortoilíaco, la hipertensión no tuvo relación para la enfermedad en este segmento.

En el 2006, Diehm, et al. realizaron un estudio de cohortes cuyo objetivo fue correlacionar el patrón de distribución de aterosclerosis de los miembros inferiores con el perfil de riesgo cardiovascular de los pacientes con EAP; este protocolo incluyó a una serie de 2,659 pacientes y se realizó un análisis de regresión logística multivariable. Se encontró que el tabaquismo era un factor predisponente para enfermedad aortoilíaca (RRR, 2.02; IC95\%, 1.68-2.42) y el sexo masculino también tuvo una significancia estadística (RRR, 1.32, 1.09-1.59) a diferencia de lo mostrado en este estudio que no halló significancia estadística por género, lo cual puede deberse a que en la actualidad el sexo femenino muestra un incremento del tabaquismo ${ }^{15}$. En cuanto al segmento femoropoplíteo, los resultados son similares a los resultados de los autores. $Y$ en el segmento 
Tabla 4. Modelo de regresión logística para el desarrollo de enfermedad femoropoplítea y enfermedad aortoilíaca entre pacientes diabéticos 0 con otro factor de riesgo cardiovascular estudiados y atendidos en la UMAE 71 en el periodo de enero de 2018 a marzo de 2020

\begin{tabular}{|c|c|c|c|c|}
\hline \multicolumn{5}{|c|}{ Enfermedad femoropoplítea } \\
\hline \multirow[t]{2}{*}{ Variable } & \multirow[t]{2}{*}{ B } & \multirow[t]{2}{*}{ OR ajustado } & \multicolumn{2}{|c|}{ IC95\% } \\
\hline & & & Mínimo & Máximo \\
\hline Hombres & -0.763 & 0.466 & 0.253 & 0.862 \\
\hline Tabaquismo & -0.115 & 0.892 & 0.480 & 1.657 \\
\hline Hipertensión arterial & -0.880 & 0.415 & 0.180 & 0.956 \\
\hline Enfermedad renal crónica & -0.371 & 0.690 & 0.343 & 1.388 \\
\hline Cardiopatía isquémica & 0.020 & 1.021 & 0.462 & 2.256 \\
\hline Diabetes mellitus & 0.231 & 1.260 & 0.585 & 2.711 \\
\hline \multicolumn{5}{|c|}{ Enfermedad aortoilíaca } \\
\hline \multirow[t]{2}{*}{ Variable } & \multirow[t]{2}{*}{ B } & \multirow[t]{2}{*}{ OR ajustado } & \multicolumn{2}{|c|}{ IC95\% } \\
\hline & & & Mínimo & Máximo \\
\hline Hombres & 0.367 & 1.444 & 0.611 & 3.415 \\
\hline Tabaquismo & 1.304 & 3.685 & 1.346 & 10.087 \\
\hline Hipertensión arterial & -1.945 & 0.143 & 0.061 & 0.334 \\
\hline Enfermedad renal crónica & 0.367 & 1.443 & 0.571 & 3.649 \\
\hline Cardiopatía isquémica & -1.656 & 0.191 & 0.024 & 1.540 \\
\hline Diabetes mellitus & -0.652 & 0.521 & 0.174 & 1.560 \\
\hline
\end{tabular}

infragenicular se registró significancia estadística para diabetes, al igual que los resultados propios y para el sexo masculino (RRR, 1.23, 1.06-1.41), lo cual no tuvo este estudio significancia estadística.

Uno de los estudios más recientes sobre la distribución de la enfermedad aterosclerótica en diabéticos lo publicaron Lowry, et al. en 2018 y demostró que los pacientes con DM tenían significativamente menos probabilidades de sufrir enfermedad en el segmento aortoilíaco (OR, 0.25 [0.15-0.42], n = DM 466/NDM 458) y significativamente más probabilidades de desarrollar enfermedad en el segmento de vasos tibiales (OR, 1.94 1.27-2.96]; $n=$ DM 306/NDM 417) ${ }^{16}$. Estos datos son similares a los obtenidos en el estudio de los autores.

Se dispone de evidencia según la cual la DM ejerce un efecto en la presentación de la enfermedad arterial crónica, en particular en el segmento infragenicular, y en los resultados después de la revascularización ${ }^{17}$. La fisiopatología que subyace a la relación entre DM y EAP (enfermedad arterial periférica crónica) es compleja; en estudios recientes se ha demostrado que múltiples aberraciones metabólicas en la diabetes mellitus, como los productos finales de la glucación avanzada, el colesterol de lipoproteínas de baja densidad y el estrés oxidativo anormal, empeoran a ésta, pero el papel de la DM en la EAP no se comprende del todo y hoy en día no hay ninguna evidencia clara que dilucide por qué los vasos distales están afectados de manera predominante ${ }^{18}$.

Estos hallazgos demuestran la relevancia clínica de la DM en el segmento de los vasos tibiales como factor principal de relación, por lo cual son importantes estudios enfocados en el papel fisiopatológico de la diabetes en este segmento para valorar el efecto de los tratamientos médicos y quirúrgicos. Otro hallazgo sobresaliente es la hipertensión como factor de riesgo de menor relación; es esencial investigar la hemodinamia del flujo arterial en hipertensos y su relación con las lesiones ateroscleróticas con un tamaño de muestra mayor.

Como limitación de este estudio, no fue posible realizar un análisis individual de cada vaso tibial debido a que no se proporcionaba específicamente 
en el expediente clínico (hoja posquirúrgica) la información necesaria; además, no fue posible valorar todas las arteriografías, ya que algunas no se encontraban en la base de datos de hemodinamia y fue necesario basarse en el informe posquirúrgico.

\section{Conclusión}

Los pacientes con DM tienen mayor relación para enfermedades de vasos tibiales en comparación con los pacientes no diabéticos. Es preciso comprender en futuros protocolos por qué los vasos distales están más afectados en diabéticos para determinar el efecto del tratamiento médico y quirúrgico (abierto/endovascular) y con ello mejorar los resultados clínicos en esta población que se halla en crecimiento, no sólo en México sino en el mundo entero.

Encuanto a los otros segmentos estudiados, la enfermedad $A O$ se vincula con el tabaquismo y la enfermedad FP con el género femenino; estos datos se relacionan con los informes publicados en la actualidad. La hipertensión en lesiones en estos segmentos en esta población (AO y FP) tiene una menor relación.

\section{Agradecimientos}

A mis padres, Enrique y Regina, por ser el motor que me impulsa a crecer cada día.

Al Instituto Mexicano del Seguro Social, por darme lo necesario para crecer profesionalmente.

\section{Conflicto de intereses}

Los autores declaran no tener conflicto de intereses alguno.

\section{Financiamiento}

Esta investigación no ha recibido ninguna beca específica de instituciones de los sectores públicos, comercial, o sin ánimo de lucro.

\section{Responsabilidades éticas}

Protección de personas y animales. Los autores declaran que para esta investigación no se han realizado experimentos en seres humanos ni en animales.

Confidencialidad de los datos. Los autores declaran que han seguido los protocolos de su centro de trabajo sobre la publicación de datos de pacientes.

Derecho a la privacidad y consentimiento informado. Los autores declaran que en este artículo no aparecen datos de pacientes.

\section{Bibliografía}

1. Kullo IJ, Rooke TW. Peripheral artery disease. $N$ Engl J Med. 2016;374(9):861-871.

2. Chen $Q$, Shi Y, Wang Y. Patterns of disease distribution of lower extremity peripheral arterial disease. 2015;66(3):211-218

3. Urbano L, Portilla E, Mu W. Prevalence and risk factors associated with peripheral arterial disease in an adult population from Colombia. 2018;88(2).

4. Conte MS. Clinical practice guideline document global vascular guidelines on the management of chronic limb-threatening ischemia. Eur J Vasc Endovasc Surg. 2019;58:1-109.

5. Criqui $\mathrm{MH}$, Aboyans V. Epidemiology of peripheral artery disease. Circ Res. 2015;116(9):1509-1526.

6. Fowkes FGR. Comparison of global estimates of prevalence and risk factors for peripheral artery disease in 2000 and 2010: a systematic review and analysis. Lancet. 2013;382(9901):1329-1340.

7. Heart disease in Latin America risk factors for acute myocardial infarction. 2007.

8. Hernando FJS, Conejero AM. Peripheral artery disease: pathophysiology, diagnosis and treatment. Rev Esp Cardiol. 2007;60(9):969-982.

9. Krishna SM, Moxon JV, Golledge J. A review of the pathophysiology and potential biomarkers for peripheral artery disease. Int $\mathrm{J}$ Mol Sci. 2015;16(5):11294-11322.

10. Fowkes FGR, Aboyans V, Fowkes FJI, McDermott MM, ampson UKA, Criqui $\mathrm{MH}$. Peripheral artery disease: epidemiology and global perspectives. Nat Rev Cardiol. 2017:;14(3):156-170.

11. Akalu Y. Peripheral arterial disease and its associated factors among type 2 diabetes mellitus patients at Debre Tabor General Hospital, Northwest Ethiopia. 2020;2020.

12. Guariguata L, Whiting DR, Hambleton I, Beagley J. Global estimates of diabetes prevalence for 2013 and projections for 2035. Diabetes Res Clin Pract. 2013;103(2):137-149.

13. Sidawy AN, Perler BA. Rutherford's vascular surgery and endovascular therapy 9 th. 2019

14. Hinojosa Becerril CA, Anaya Ayala JE, Laparra Escareño H. Acciones a favor de los accesos vasculares para hemodiálisis en México. 2019.

15. Conen D. Smoking, smoking status, and risk for symptomatic peripheral artery disease in women. Ann Intern Med. 2011;154(11):719-726.

16. Lowry D, Saeed M, Uk M, Uk F, Narendran P, Tiwari A. A review of distribution of atherosclerosis in the lower limb arteries of patients with diabetes mellitus and peripheral vascular disease. 2018.

17. Abularrage CJ. Long-term outcomes of diabetic patients undergoing endovascular infrainguinal interventions. YMVA. 2007;52(2):314-322.e4.

18. Yang S, Zhu L, Han R, Sun L, Li J, Dou JT. Pathophysiology of peripheral arterial disease in diabetes mellitus. 